\title{
LABORATORIES FOR CEMENT RESEARCH
}

$\mathrm{T}$ HE new research laboratories of the Associated Portland Cement Manufacturers, Ltd., at Stone, in Kent, were opened on July 11 by the Minister of Works, the Right Hon. D. McA. Eccles. These laboratories, together with general offices, form a pleasant building, and the architects, Messrs. Westwood, Sons and Harrison, are to be congratulated on the effect of modestly decorating a functional building with Tyrolean and coloured finishes and by the occasional use of Kentish rag-stone.

In these laboratories and offices, a large staff of well-qualified scientific men and technicians will work towards the improvement of the quality of cement, the cheapening of production, and the introduction of new products, in addition to dealing with specific problems which continually arise. Engineering research in connexion with cement-making plant will form an important part of the work, as well as fundamental research in the chemistry and physics of cement manufacture, and its behaviour in the making and curing of concrete and many allied materials. The development and improvement of methods of manufacturing burnt clay, sulphate-resisting and low-heat cement are also important objects.

'I $\mathrm{T}$ the laboratories are well equipped. The lay-out of the chemical laboratories is of particular interest. Artificial ventilation effectively disposes of fumes, and subsidiary side-rooms are provided to house such equipment as muffles, furnaces, hot plates, water-baths and additional fume cupboards, etc. The Physical Testing Department has the usual equipment for cement and concrete testing, including temperature- and humidity-controlled curing rooms. It is evident from results that when testing is carried

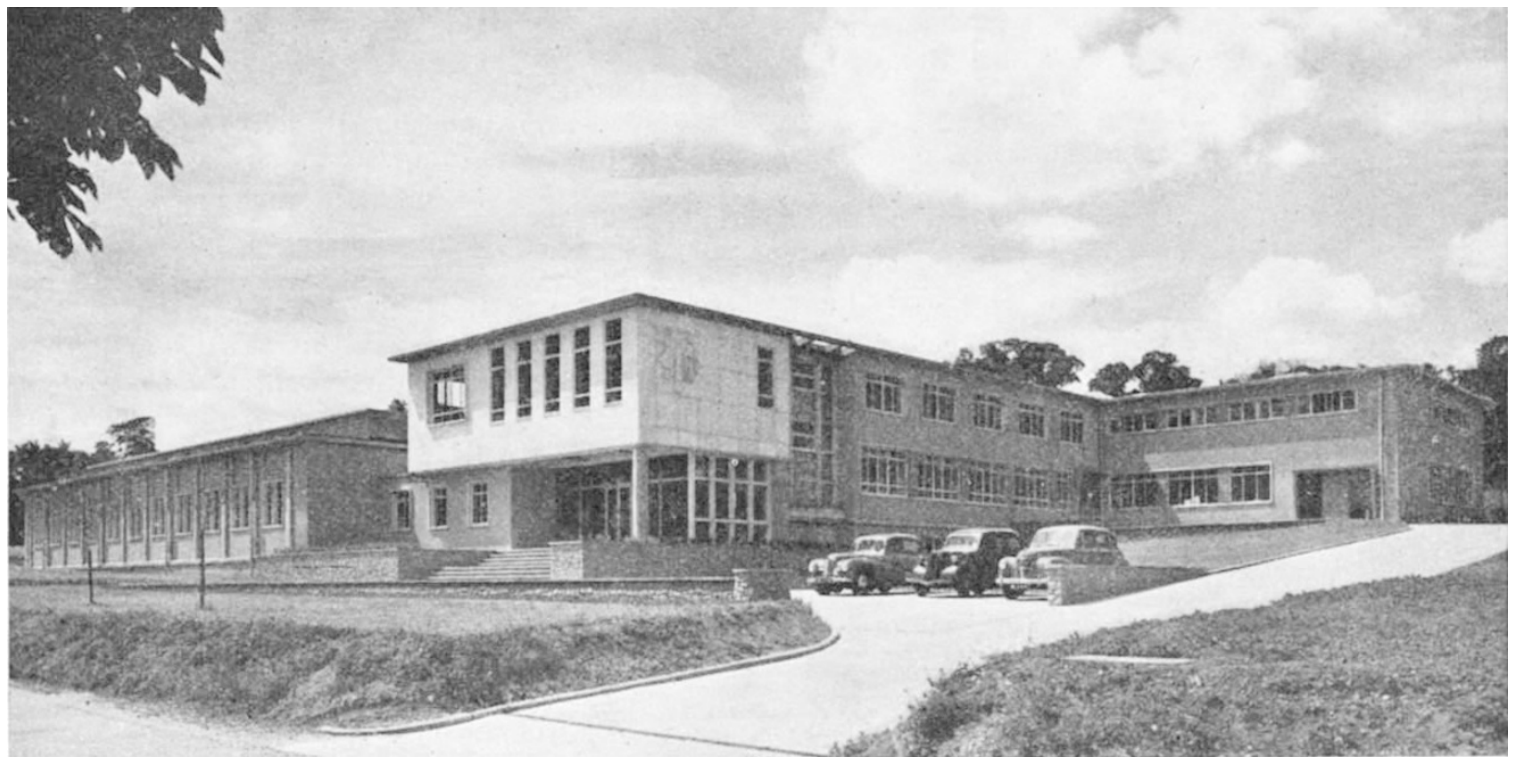

Fig. 1. Research Laboratories of the Associated Portland Cement Manufacturers, Ltd., at Stone

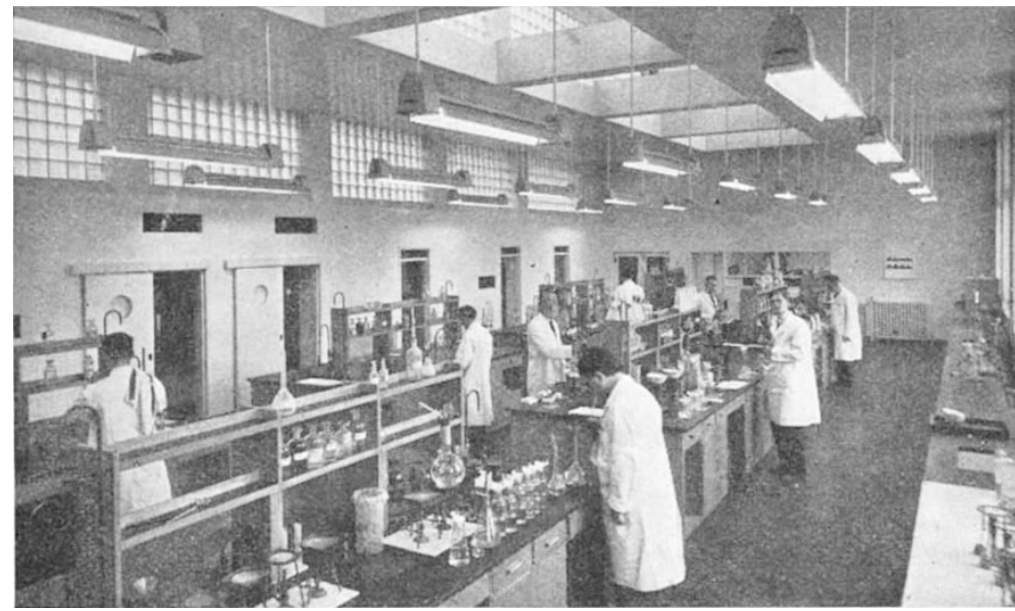

Fig. 2. Chemical Laboratories out under rigorously controlled conditions the uniformity of strength of cubes is as good as can be desired when made from the same batches of cement.

It would take too much space to describe in detail all departments ; but it should be mentioned that in the laboratories there is a Kilns and Mills Division, and a Raw Maverials Section, both well equipped and doing important work. There is a Microscopic Department, and a Library is beginning to accumulate. 'lhere is a lecture room in which instruction is given to personnel undergoing training in cement technology. This activity is considered to be one of the most important functions of the laboratory. A large and pleasant canteen has been 
provided ; in fact, the visitor receives the impression that every aspect of planning has been well considered.

The staff of the laboratory will no doubt contribute greatly in the future towards improvements in the cement industry, and so give further help towards the solution of some of our economic problems, to which the cement industry has already contributed much.

\section{GERMAN SOCIETY FOR ELECTRON MICROSCOPY}

\section{FOURTH ANNUAL CONFERENCE}

$\mathrm{T}$

HE fourth annual conference of the German Society for Electron Microscopy took place this year at Tübingen during June 6-9. The increasing interest evoked by this event, both inside and outside Germany, may be judged by the attendance at the conference of more than two hundred electron microscopists, many of whom came from abroad. Although this was not an international conference, eight European countries were, in fact, represented. A high standard was maintained both in the papers presented and in the discussions. It was felt, however, that the total number of eighty papers was rather large considering the limited time available. It is only possible to mention a few of the papers here, and these have been chosen so far as possible to indicate the general scope of the conference.

The chairman of the Society, Prof. U. Hofmann, delivered the opening lecture, choosing as his subject "The Electron Microscope in the Treatment of Problems in Inorganic Chemistry". The electron microscope, he said, has made its contribution to organic chemistry largely by virtue of its ability to resolve fine structures, particularly those of crystals. It has been used, for example, for determining the structure of graphite crystals in connexion with problems of lubrication. Similarly, with its aid, progress has been made in the chemistry of soils and clays and in the selection of suitable fillers for rubbers. Although the resolving power of the electron microscope is not yet sufficient to resolve atomic structure, it is often possible to use the instrument for obtaining an estimate of surface area and crystal size, thus obtaining a valuable check on $\mathrm{X}$-ray determinations from line-broadening measurements.

Several papers were devoted to the imaging properties of electron lenses. H. Bremmer developed a simple approximate formula for the axial field distribution in a magnetic lens in terms of the lens excitation and pole-piece geometry. S. Leisegang presented a theoretical paper on the form of the caustics arising in lenses suffering from astigmatism. Previous work by W. Glaser and E. Regenstreif was reviewed, and a number of caustic cross-sections were shown arising from different types of field asymmetry. By observing such caustics in a lens provided with a suitable correcting device, it is possible in principle to compensate the lens astigmatism.

F. Lenz and M. Hahn showed how this could be done experimentally. Electrons are arranged to travel through an astigmatic lens in slightly undertelescopic ray-paths. The emerging electrons strike a distant fluorescent screen forming a pattern there, consisting of an astroid surrounded by a circle. Using Sturrock's theory of the astigmatic lens, Lenz and Hahn have correlated quantitatively the dimensions of this pattern with the asymmetries of the pole-piece system. Experimental results were shown for a particular magnetic lens in which the astigmatic difference of foci goes through a minimum value as the lens excitation is increased from zero to the maximum value available.

B. Von Borries, F. Lenz and G. Opfer described some experimental work undertaken to resolve a discrepancy amounting to 10 per cent between the focal lengths of a magnetic objective lens as calculated by Lenz and those measured by E. Ruska. Ruska's measurements were repeated and found correct. Further experiments on the magnetic circuit showed that, due to the design of the iron casing of the lens and to the $B / H$ properties of the iron used, a loss of magnetomotive force of 5 per cent occurred, causing an increase in focal length of 10 per cent above the calculated value. This accounted for the discrepancy.

R. Seeliger reported progress in the attempts to construct an electron microscope in which the primary spherical aberration is eliminated using correction methods proposed by Scherzer. The mechanical and electrical stability of the instrument has been considerably improved, and the complete correction of primary spherical aberration has been realized. There are, however, many practical difficulties still to be overcome in connexion with the stability of the specimen, etc., before the greatly increased theoretical resolving power of the lens system can be fully attained.

A new approach to the problem of reflexion specimens for the electron microscope was put forward by G. Möllenstedt and H. Düker. Measurements of the velocity distribution of secondary electrons leaving a surface which is subjected to ion bombardment showed that the energy spread was only $3 \mathrm{eV}$. In the new microscope a metal specimen is placed at right angles to the optical axis and is bombarded with low-voltage ions by means of an ion source mounted at an angle of $45^{\circ}$ to the specimen. The resulting secondary electrons are imaged at the fluorescent screen by an electrostatic lens placed immediately below the specimen. An accelerating voltage of $30 \mathrm{kV}$. is employed. The image has the appearance of that due to a shadowed specimen owing to the inclination of the ion source, thus facilitating the interpretation of the pictures. The resolution is at present about $1000 \mathrm{~A}$.; but it is believed that this can be improved considerably. The defects associated with previous systems employing glancing incidence at the specimen, namely, gross distortion of the field and high chromatic aberration, are avoided.

L. Wegmann outlined the design of a new TrübTäuber electron microscope which embodies several novel constructional features. An electrostatic objective and first projector lens are used, only the final projector being magnetic. The lenses are mounted on a hinged door provided with a vacuum seal. This construction makes the lenses readily accessible. In addition, the projector lenses are mounted on bearings so that they may be swung out of the path of the beam when a direct diffraction pattern is required. Two ranges of magnification are provided : 800-4,000 for comparison work with the optical microscope, and 8,000-14,000. A focusing aid is incorporated which causes three images of each object point to appear when the instrument is incorrectly focused. This is achieved by providing two extra-axial holes in the condenser aperture in 